\title{
The tripartite separability of density matrices of graphs
}

\author{
Zhen Wang and Zhixi Wang \\ Department of Mathematics \\ Capital Normal University, Beijing 100037, China \\ wangzhen061213@sina.com, wangzhx@mail.cnu.edu.cn \\ Submitted: May 9, 2007; Accepted: May 16, 2007; Published: May 23, 2007 \\ Mathematics Subject Classification: 81P15
}

\begin{abstract}
The density matrix of a graph is the combinatorial laplacian matrix of a graph normalized to have unit trace. In this paper we generalize the entanglement properties of mixed density matrices from combinatorial laplacian matrices of graphs discussed in Braunstein et al. [Annals of Combinatorics, 10 (2006) 291] to tripartite states. Then we prove that the degree condition defined in Braunstein et al. [Phys. Rev. A, 73 (2006) 012320] is sufficient and necessary for the tripartite separability of the density matrix of a nearest point graph.
\end{abstract}

\section{Introduction}

Quantum entanglement is one of the most striking features of the quantum formalism ${ }^{[1]}$. Moreover, quantum entangled states may be used as basic resources in quantum information processing and communication, such as quantum cryptography ${ }^{[2]}$, quantum parallelism $^{[3]}$, quantum dense coding ${ }^{[4,5]}$ and quantum teleportation ${ }^{[6,7]}$. So testing whether a given state of a composite quantum system is separable or entangled is in general very important.

Recently, normalized laplacian matrices of graphs considered as density matrices have been studied in quantum mechanics. One can recall the definition of density matrices of graphs from [8]. Ali Saif M. Hassan and Pramod Joag ${ }^{[9]}$ studied the related issues like classification of pure and mixed states, von Neumann entropy, separability of multipartite quantum states and quantum operations in terms of the graphs associated with quantum states. Chai Wah $\mathrm{Wu}^{[10]}$ showed that the Peres-Horodecki positive partial transpose condition is necessary and sufficient for separability in $C^{2} \otimes C^{q}$. Braunstein et al. ${ }^{[11]}$ proved that the degree condition is necessary for separability of density matrices of any graph and is sufficient for separability of density matrices of nearest point graphs and perfect 
matching graphs. Hildebrand et al. ${ }^{[12]}$ testified that the degree condition is equivalent to the PPT-criterion. They also considered the concurrence of density matrices of graphs and pointed out that there are examples on four vertices whose concurrence is a rational number.

The paper is divided into three sections. In section 2, we recall the definition of the density matrices of a graph and define the tensor product of three graphs, reconsider the tripartite entanglement properties of the density matrices of graphs introduced in [8]. In section 3, we define partially transposed graph at first and then shows that the degree condition introduced in [11] is also sufficient and necessary condition for the tripartite state of the density matrices of nearest point graphs.

\section{The tripartite entanglement properties of the den- sity matrices of graphs}

Recall that from [8] a graph $G=(V(G), E(G))$ is defined as: $V(G)=\left\{v_{1}, v_{2}, \cdots, v_{n}\right\}$ is a non-empty and finite set called vertices; $E(G)=\left\{\left\{v_{i}, v_{j}\right\}: v_{i}, v_{j} \in V\right\}$ is a nonempty set of unordered pairs of vertices called edges. An edge of the form $\left\{v_{i}, v_{i}\right\}$ is called as a loop. We assume that $E(G)$ does not contain any loops. A graph $G$ is said to be on $n$ vertices if $|V(G)|=n$. The adjacency matrix of a graph $G$ on $n$ vertices is an $n \times n$ matrix, denoted by $M(G)$, with lines labeled by the vertices of $G$ and $i j$-th entry defined as:

$$
[M(G)]_{i, j}= \begin{cases}1, & \text { if }\left(v_{i}, v_{j}\right) \in E(G) \\ 0, & \text { if }\left(v_{i}, v_{j}\right) \notin E(G)\end{cases}
$$

If $\left\{v_{i}, v_{j}\right\} \in E(G)$ two distinct vertices $v_{i}$ and $v_{j}$ are said to be adjacent. The degree of a vertex $v_{i} \in V(G)$ is the number of edges adjacent to $v_{i}$, we denote it as $d_{G}\left(v_{i}\right)$. $d_{G}=\sum_{i=1}^{n} d_{G}\left(v_{i}\right)$ is called as the degree sum. Notice that $d_{G}=2|E(G)|$. The degree matrix of $G$ is an $n \times n$ matrix, denoted as $\Delta(G)$, with $i j$-th entry defined as:

$$
[\Delta(G)]_{i, j}= \begin{cases}d_{G}\left(v_{i}\right), & \text { if } i=j \\ 0, & \text { if } i \neq j\end{cases}
$$

The combinatorial laplacian matrix of a graph $G$ is the symmetric positive semidefinite matrix

$$
L(G)=\Delta(G)-M(G) .
$$

The density matrix of $G$ of a graph $G$ is the matrix

$$
\rho(G)=\frac{1}{d_{G}} L(G)
$$

Recall that a graph is called complete $e^{[14]}$ if every pair of vertices are adjacent, and the complete graph on $n$ vertices is denoted by $K_{n}$. Obviously, $\rho\left(K_{n}\right)=\frac{1}{n(n-1)}\left(n I_{n}-J_{n}\right)$, 
where $I_{n}$ and $J_{n}$ is the $n \times n$ identity matrix and the $n \times n$ all-ones matrix, respectively. A star graph on $n$ vertices $\alpha_{1}, \alpha_{2}, \cdots, \alpha_{n}$, denoted by $K_{1, n-1}$, is the graph whose set of edges is $\left\{\left\{\alpha_{1}, \alpha_{i}\right\}: i=2,3, \cdots, n\right\}$, we have

$$
\rho\left(K_{1, n-1}\right)=\frac{1}{2(n-1)}\left(\begin{array}{ccccc}
n-1 & -1 & -1 & \cdots & -1 \\
-1 & 1 & & & \\
-1 & & 1 & & \\
\vdots & & & \ddots & \\
-1 & & & & 1
\end{array}\right) .
$$

Let $G$ be a graph which has only a edge. Then the density matrix of $G$ is pure. The density matrix of a graph is a uniform mixture of pure density matrices, that is, for a graph $G$ on $n$ vertices $v_{1}, v_{2}, \cdots, v_{n}$, having $s$ edges $\left\{v_{i_{1}}, v_{j_{1}}\right\},\left\{v_{i_{2}}, v_{j_{2}}\right\}, \cdots,\left\{v_{i_{s}}, v_{j_{s}}\right\}$, where $1 \leq i_{1}, j_{1}, i_{2}, j_{2}, \cdots, i_{k}, j_{k} \leq n$,

$$
\rho(G)=\frac{1}{s} \sum_{k=1}^{s} \rho\left(H_{i_{k} j_{k}}\right)
$$

here $H_{i_{k} j_{k}}$ is the factor of $G$ such that

$$
\left[M\left(H_{i_{k} j_{k}}\right)\right]_{u, w}= \begin{cases}1, & \text { if } u=i_{k} \text { and } w=j_{k} \text { or } w=i_{k} \text { and } u=j_{k} \\ 0, & \text { otherwise. }\end{cases}
$$

It is obvious that $\rho\left(H_{i_{k} j_{k}}\right)$ is pure.

Before we discuss the tripartite entanglement properties of the density matrices of graphs we will at first recall briefly the definition of the tripartite separability:

Definition 1 The state $\rho$ acting on $\mathcal{H}=\mathcal{H}_{\mathcal{A}} \otimes \mathcal{H}_{\mathcal{B}} \otimes \mathcal{H}_{\mathcal{C}}$ is called tripartite separability if it can be written in the form

$$
\rho=\sum_{i} p_{i} \rho_{A}^{i} \otimes \rho_{B}^{i} \otimes \rho_{C}^{i}
$$

where $\rho_{A}^{i}=\left|\alpha_{A}^{i}\right\rangle\left\langle\alpha_{A}^{i}\left|, \rho_{B}^{i}=\right| \beta_{B}^{i}\right\rangle\left\langle\beta_{B}^{i}\left|, \rho_{C}^{i}=\right| \gamma_{C}^{i}\right\rangle\left\langle\gamma_{C}^{i}\right|, \sum_{i} p_{i}=1, p_{i} \geq 0$ and $\left|\alpha_{A}^{i}\right\rangle,\left|\beta_{B}^{i}\right\rangle$, $\left|\gamma_{C}^{i}\right\rangle$ are normalized pure states of subsystems $A, B$ and $C$, respectively. Otherwise, the state is called entangled.

Now we define the tensor product of three graphs. The tensor product of graphs $G_{A}, G_{B}, G_{C}$, denoted by $G_{A} \otimes G_{B} \otimes G_{C}$, is the graph whose adjacency matrix is $M\left(G_{A} \otimes G_{B} \otimes G_{C}\right)=M\left(G_{A}\right) \otimes M\left(G_{B}\right) \otimes M\left(G_{C}\right)$. Whenever we consider a graph $G_{A} \otimes G_{B} \otimes G_{C}$, where $G_{A}$ is on $m$ vertices, $G_{B}$ is on $p$ vertices and $G_{C}$ is on $q$ vertices, the tripartite separability of $\rho\left(G_{A} \otimes G_{B} \otimes G_{C}\right)$ is described with respect to the Hilbert space $\mathcal{H}_{A} \otimes \mathcal{H}_{B} \otimes \mathcal{H}_{C}$, where $\mathcal{H}_{A}$ is the space spanned by the orthonormal basis 
$\left\{\left|u_{1}\right\rangle,\left|u_{2}\right\rangle, \cdots,\left|u_{m}\right\rangle\right\}$ associated to $V\left(G_{A}\right), \mathcal{H}_{B}$ is the space spanned by the orthonormal basis $\left\{\left|v_{1}\right\rangle,\left|v_{2}\right\rangle, \cdots,\left|v_{p}\right\rangle\right\}$ associated to $V\left(G_{B}\right)$ and $\mathcal{H}_{C}$ is the space spanned by the orthonormal basis $\left\{\left|w_{1}\right\rangle,\left|w_{2}\right\rangle, \cdots,\left|w_{q}\right\rangle\right\}$ associated to $V\left(G_{C}\right)$. The vertices of $G_{A} \otimes G_{B} \otimes G_{C}$ are taken as $\left\{u_{i} v_{j} w_{k}, 1 \leq i \leq m, 1 \leq j \leq p, 1 \leq k \leq q\right\}$. We associate $\left|u_{i}\right\rangle\left|v_{j}\right\rangle\left|w_{k}\right\rangle$ to $u_{i} v_{j} w_{k}$, where $1 \leq i \leq m, 1 \leq j \leq p, 1 \leq k \leq q$. In conjunction with this, whenever we talk about tripartite separability of any graph $G$ on $n$ vertices, $\left|\alpha_{1}\right\rangle,\left|\alpha_{2}\right\rangle, \cdots,\left|\alpha_{n}\right\rangle$, we consider it in the space $C^{m} \otimes C^{p} \otimes C^{q}$, where $n=m p q$. The vectors $\left|\alpha_{1}\right\rangle,\left|\alpha_{2}\right\rangle, \cdots,\left|\alpha_{n}\right\rangle$ are taken as follows: $\left|\alpha_{1}\right\rangle=\left|u_{1}\right\rangle\left|v_{1}\right\rangle\left|w_{1}\right\rangle,\left|\alpha_{2}\right\rangle=$ $\left|u_{1}\right\rangle\left|v_{1}\right\rangle\left|w_{2}\right\rangle, \cdots,\left|\alpha_{n}\right\rangle=\left|u_{m}\right\rangle\left|v_{p}\right\rangle\left|w_{q}\right\rangle$.

To investigate the tripartite entanglement properties of the density matrices of graphs it is necessary to recall the well known positive partial transposition criterion (i.e. Peres criterion). It makes use of the notion of partial transpose of a density matrix. Here we will only recall the Peres criterion for the tripartite states. Consider a $n \times n$ matrix $\rho_{A B C}$ acting on $C_{A}^{m} \otimes C_{B}^{p} \otimes C_{C}^{q}$, where $n=m p q$. The partial transpose of $\rho_{A B C}$ with respect to the systems $A, B, C$ are the matrices $\rho_{A B C}^{T_{A}}, \rho_{A B C}^{T_{B}}, \rho_{A B C}^{T_{C}}$, respectively, and with $\left(i, j, k ; i^{\prime}, j^{\prime}, k^{\prime}\right)$-th entry defined as follows:

$$
\begin{aligned}
& {\left[\rho_{A B C}^{T_{A}}\right]_{i, j, k ; i^{\prime}, j^{\prime}, k^{\prime}}=\left\langle u_{i^{\prime}} v_{j} w_{k}\left|\rho_{A B C}\right| u_{i} v_{j^{\prime}} w_{k^{\prime}}\right\rangle,} \\
& {\left[\rho_{A B C}^{T_{B}}\right]_{i, j, k ; i^{\prime}, j^{\prime}, k^{\prime}}=\left\langle u_{i} v_{j^{\prime}} w_{k}\left|\rho_{A B C}\right| u_{i^{\prime}} v_{j} w_{k^{\prime}}\right\rangle,} \\
& {\left[\rho_{A B C}^{T_{C}}\right]_{i, j, k ; i^{\prime}, j^{\prime}, k^{\prime}}=\left\langle u_{i} v_{j} w_{k^{\prime}}\left|\rho_{A B C}\right| u_{i^{\prime}} v_{j^{\prime}} w_{k}\right\rangle,}
\end{aligned}
$$

where $1 \leq i, i^{\prime} \leq m ; 1 \leq j, j^{\prime} \leq p$ and $1 \leq k, k^{\prime} \leq q$.

For separability of $\rho_{A B C}$ we have the following criterion:

Peres criterion $^{[13]}$ If $\rho$ is a separable density matrix acting on $C^{m} \otimes C^{p} \otimes C^{q}$, then $\rho^{T_{A}}, \rho^{T_{B}}, \rho^{T_{C}}$ are positive semidefinite.

Lemma 1 The density matrix of the tensor product of three graphs is tripartite separable.

Proof. Let $G_{1}$ be a graph on $n$ vertices, $u_{1}, u_{2}, \cdots, u_{n}$, and $m$ edges, $\left\{u_{c_{1}}\right.$, $\left.u_{d_{1}}\right\}, \cdots,\left\{u_{c_{m}}, u_{d_{m}}\right\}, 1 \leq c_{1}, d_{1}, \cdots, c_{m}, d_{m} \leq n$. Let $G_{2}$ be a graph on $k$ vertices, $v_{1}, v_{2}, \cdots, v_{k}$, and $e$ edges, $\left\{v_{i_{1}}, v_{j_{1}}\right\}, \cdots,\left\{v_{i_{e}}, v_{j_{e}}\right\}, 1 \leq i_{1}, j_{1}, \cdots, i_{e}, j_{e} \leq k$. Let $G_{3}$ be a graph on $l$ vertices, $w_{1}, w_{2}, \cdots, w_{l}$, and $f$ edges, $\left\{w_{r_{1}}, w_{s_{1}}\right\}, \cdots,\left\{w_{r_{f}}, w_{s_{f}}\right\}, 1 \leq$ $r_{1}, s_{1}, \cdots, r_{f}, s_{f} \leq l$. Then

$$
\rho\left(G_{1}\right)=\frac{1}{m} \sum_{p=1}^{m} \rho\left(H_{c_{p} d_{p}}\right), \rho\left(G_{2}\right)=\frac{1}{e} \sum_{q=1}^{e} \rho\left(L_{i_{q} j_{q}}\right), \rho\left(G_{3}\right)=\frac{1}{f} \sum_{t=1}^{f} \rho\left(Q_{r_{t} s_{t}}\right) .
$$

Therefore

$$
\begin{aligned}
\rho\left(G_{1}\right. & \left.\otimes G_{2} \otimes G_{3}\right) \\
& =\frac{1}{d_{G_{1} \otimes G_{2} \otimes G_{3}}}\left[\Delta\left(G_{1} \otimes G_{2} \otimes G_{3}\right)-M\left(G_{1} \otimes G_{2} \otimes G_{3}\right)\right]
\end{aligned}
$$




$$
\begin{aligned}
= & \frac{1}{d_{G_{1} \otimes G_{2} \otimes G_{3}}} \sum_{p=1}^{m} \sum_{q=1}^{e} \sum_{t=1}^{f}\left[\Delta\left(H_{c_{p} d_{p}} \otimes L_{i_{q} j_{q}} \otimes Q_{r_{t} s_{t}}\right)-M\left(H_{c_{p} d_{p}} \otimes L_{i_{q} j_{q}} \otimes Q_{r_{t} s_{t}}\right)\right] \\
= & \frac{1}{d_{G_{1} \otimes G_{2} \otimes G_{3}}} \sum_{p=1}^{m} \sum_{q=1}^{e} \sum_{t=1}^{f} 8 \rho\left(H_{c_{p} d_{p}} \otimes L_{i_{q} j_{q}} \otimes Q_{r_{t} s_{t}}\right) \\
= & \frac{1}{m e f} \sum_{p=1}^{m} \sum_{q=1}^{e} \sum_{t=1}^{f} \rho\left(H_{c_{p} d_{p}} \otimes L_{i_{q} j_{q}} \otimes Q_{r_{t} s_{t}}\right) \\
= & \frac{1}{m e f} \sum_{p=1}^{m} \sum_{q=1}^{e} \sum_{t=1}^{f} \frac{1}{8}\left[\Delta\left(H_{c_{p} d_{p}}\right) \otimes \Delta\left(L_{i_{q} j_{q}}\right) \otimes \Delta\left(Q_{r_{t} s_{t}}\right)-M\left(H_{c_{p} d_{p}}\right) \otimes M\left(L_{i_{q} j_{q}}\right) \otimes M\left(Q_{r_{t} s_{t}}\right)\right] \\
= & \frac{1}{m e f} \sum_{p=1}^{m} \sum_{q=1}^{e} \sum_{t=1}^{f} \frac{1}{4}\left[\rho\left(H_{c_{p} d_{p}}\right) \otimes \rho\left(L_{i_{q} j_{q}}\right) \otimes \rho\left(Q_{r_{t} s_{t}}\right)\right. \\
& +\rho_{+}\left(H_{c_{p} d_{p}}\right) \otimes \rho\left(L_{i_{q} j_{q}}\right) \otimes \rho_{+}\left(Q_{r_{t} s_{t}}\right)+\rho\left(H_{c_{p} d_{p}}\right) \otimes \rho_{+}\left(L_{i_{q} j_{q}}\right) \otimes \rho_{+}\left(Q_{r_{t} s_{t}}\right) \\
& \left.+\rho_{+}\left(H_{c_{p} d_{p}}\right) \otimes \rho_{+}\left(L_{i_{q} j_{q}}\right) \otimes \rho\left(Q_{r_{t} s_{t}}\right)\right]
\end{aligned}
$$

where

$$
\begin{aligned}
& \rho_{+}\left(H_{c_{p} d_{p}}\right) \stackrel{\text { def }}{=} \Delta\left(H_{c_{p} d_{p}}\right)-\rho\left(H_{c_{p} d_{p}}\right)=\frac{1}{2}\left(\Delta\left(H_{c_{p} d_{p}}\right)+M\left(H_{c_{p} d_{p}}\right)\right), \\
& \rho_{+}\left(L_{i_{q} j_{q}}\right) \stackrel{\text { def }}{=} \Delta\left(L_{i_{q} j_{q}}\right)-\rho\left(L_{i_{q} j_{q}}\right)=\frac{1}{2}\left(\Delta\left(L_{i_{q} j_{q}}\right)+M\left(L_{i_{q} j_{q}}\right)\right), \\
& \rho_{+}\left(Q_{r_{t} s_{t}}\right) \stackrel{\text { def }}{=} \Delta\left(Q_{r_{t} s_{t}}\right)-\rho\left(Q_{r_{t} s_{t}}\right)=\frac{1}{2}\left(\Delta\left(Q_{r_{t} s_{t}}\right)+M\left(Q_{r_{t} s_{t}}\right)\right),
\end{aligned}
$$

the fourth equality follows from $d_{G_{1} \otimes G_{2} \otimes G_{3}}=8$ mef and the fifth equality follows from the definition of tensor products of graphs.

Notice that $\rho_{+}\left(H_{c_{p} d_{p}}\right), \rho_{+}\left(L_{i_{q} j_{q}}\right), \rho_{+}\left(Q_{r_{t} s_{t}}\right)$ are all density matrices. Let

$$
\rho_{+}\left(G_{1}\right)=\frac{1}{m} \sum_{p=1}^{m} \rho_{+}\left(H_{c_{p} d_{p}}\right), \quad \rho_{+}\left(G_{2}\right)=\frac{1}{e} \sum_{q=1}^{e} \rho_{+}\left(L_{i_{q} j_{q}}\right), \quad \rho_{+}\left(G_{3}\right)=\frac{1}{f} \sum_{t=1}^{f} \rho_{+}\left(Q_{r_{t} s_{t}}\right) .
$$

Then

$$
\begin{aligned}
\rho\left(G_{1} \otimes G_{2} \otimes G_{3}\right)= & \frac{1}{4}\left[\rho\left(G_{1}\right) \otimes \rho\left(G_{2}\right) \otimes \rho\left(G_{3}\right)+\rho_{+}\left(G_{1}\right) \otimes \rho\left(G_{2}\right) \otimes \rho_{+}\left(G_{3}\right)\right. \\
& \left.+\rho\left(G_{1}\right) \otimes \rho_{+}\left(G_{2}\right) \otimes \rho_{+}\left(G_{3}\right)+\rho_{+}\left(G_{1}\right) \otimes \rho_{+}\left(G_{2}\right) \otimes \rho\left(G_{3}\right)\right] .
\end{aligned}
$$

So we have that $\rho(G)$ is tripartite separable.

Remark We associate to the vertices $\alpha_{1}, \alpha_{2}, \cdots, \alpha_{n}$ of a graph $G$ an orthonormal basis $\left\{\left|\alpha_{1}\right\rangle,\left|\alpha_{2}\right\rangle, \cdots,\left|\alpha_{n}\right\rangle\right\}$. In terms of this basis, the $u w$-th elements of the matrices $\rho\left(H_{c_{p} d_{p}}\right)$ and $\rho_{+}\left(H_{c_{p} d_{p}}\right)$ are given by $\left\langle\alpha_{u}\left|\rho\left(H_{c_{p} d_{p}}\right)\right| \alpha_{w}\right\rangle$ and $\left\langle\alpha_{u}\left|\rho_{+}\left(H_{c_{p} d_{p}}\right)\right| \alpha_{w}\right\rangle$, respectively. In this basis we have

$$
\rho\left(H_{c_{p} d_{p}}\right)=P\left[\frac{1}{\sqrt{2}}\left(\left|\alpha_{c_{p}}\right\rangle-\left|\alpha_{d_{p}}\right\rangle\right)\right], \rho_{+}\left(H_{c_{p} d_{p}}\right)=P\left[\frac{1}{\sqrt{2}}\left(\left|\alpha_{c_{p}}\right\rangle+\left|\alpha_{d_{p}}\right\rangle\right)\right] .
$$


Lemma 2 The matrix $\sigma=\frac{1}{4} P\left[\frac{1}{\sqrt{2}}(|i j k\rangle-|r s t\rangle)\right]+\frac{1}{4} P\left[\frac{1}{\sqrt{2}}(|i j t\rangle-|r s k\rangle)\right]+\frac{1}{4} P\left[\frac{1}{\sqrt{2}}(|i s k\rangle-\right.$ $|r j t\rangle)]+\frac{1}{4} P\left[\frac{1}{\sqrt{2}}(|r j k\rangle-|i s t\rangle)\right]$ is a density matrix and tripartite separable.

Proof. Since the project operator is semipositive, $\sigma$ is semipositive. By computing one can get $\operatorname{tr}(\sigma)=1$, so $\sigma$ is a density matrix. Let

$$
\left|u^{ \pm}\right\rangle=\frac{1}{\sqrt{2}}(|i\rangle \pm|r\rangle), \quad\left|v^{ \pm}\right\rangle=\frac{1}{\sqrt{2}}(|j\rangle \pm|s\rangle), \quad\left|w^{ \pm}\right\rangle=\frac{1}{\sqrt{2}}(|k\rangle \pm|t\rangle) .
$$

We obtain

$$
\sigma=\frac{1}{4} P\left[\left|u^{+}\right\rangle\left|v^{-}\right\rangle\left|w^{+}\right\rangle\right]+\frac{1}{4} P\left[\left|u^{+}\right\rangle\left|v^{+}\right\rangle\left|w^{-}\right\rangle\right]+\frac{1}{4} P\left[\left|u^{-}\right\rangle\left|v^{-}\right\rangle\left|w^{-}\right\rangle\right]+\frac{1}{4} P\left[\left|u^{-}\right\rangle\left|v^{+}\right\rangle\left|w^{+}\right\rangle\right],
$$

thus $\sigma$ is tripartite separable.

Lemma 3 For any $n=m p q$, the density matrix $\rho\left(K_{n}\right)$ is tripartite separable in $C^{m} \otimes C^{p} \otimes C^{q}$.

Proof. Since $M\left(K_{n}\right)=J_{n}-I_{n}$, where $J_{n}$ is the $n \times n$ all-ones matrix and $I_{n}$ is the $n \times n$ identity matrix, whenever there is an edge $\left\{u_{i} v_{j} w_{k}, u_{r} v_{s} w_{t}\right\}$, there must be entangled edges $\left\{u_{r} v_{j} w_{k}, u_{i} v_{s} w_{t}\right\},\left\{u_{i} v_{s} w_{k}, u_{r} v_{j} w_{t}\right\}$ and $\left\{u_{i} v_{j} w_{t}, u_{r} v_{s} w_{k}\right\}$. The result follows from Lemma 2.

Lemma 4 The complete graph on $n>1$ vertices is not a tensor product of three graphs.

Proof. It is obvious that $K_{n}$ is not a tensor product of three graphs if $n$ is a prime or a product of two primes. Thus we can assume that $n$ is a product of three or more primes. Let $n=m p q, m, p, q>1$. Suppose that there exist three graphs $G_{1}, G_{2}$ and $G_{3}$ on $m, p$ and $q$ vertices, respectively, such that $K_{m p q}=G_{1} \otimes G_{2} \otimes G_{3}$. Let $\left|E\left(G_{1}\right)\right|=$ $r,\left|E\left(G_{2}\right)\right|=s,\left|E\left(G_{3}\right)\right|=t$. Then, by the degree sum formula, $2 r \leq m(m-1), 2 s \leq$ $p(p-1), 2 t \leq q(q-1)$. Hence

$$
2 r \cdot 2 s \cdot 2 t \leq m p q(m-1)(p-1)(q-1)=m p q(m p q-m p-m q-p q+m+p+q-1) .
$$

Now, observe that

$$
\left|V\left(G_{1} \otimes G_{2} \otimes G_{3}\right)\right|=m p q, \quad\left|E\left(G_{1} \otimes G_{2} \otimes G_{3}\right)\right|=4 r s t .
$$

Therefore,

$$
G_{1} \otimes G_{2} \otimes G_{3}=K_{m p q} \Longleftrightarrow m p q(m p q-1)=2 \cdot 4 r s t
$$

so

$$
m p q(m p q-1)=8 r s t \leq m p q(m p q-m p-m q-p q+m+p+q-1) .
$$

It follows that $m p+m q+p q-m-p-q \leq 0$, that is $m(p-1)+q(m-1)+p(q-1) \leq 0$. As $m, p, q \geq 1$ we get $m(p-1)+q(m-1)+p(q-1)=0$. It yields that $m=p=q=1$.

Theorem 1 Given a graph $G_{1} \otimes G_{2} \otimes G_{3}$, the density matrix $\rho\left(G_{1} \otimes G_{2} \otimes G_{3}\right)$ is tripartite separable. However if a density matrix $\rho(L)$ is tripartite separable it does not necessarily mean that $L=L_{1} \otimes L_{2} \otimes L_{3}$, for some graphs $L_{1}, L_{2}$ and $L_{3}$.

Proof. The result follows from Lemmas 1, 3 and 4. 
Theorem 2 The density matrix $\rho\left(K_{1, n-1}\right)$ is tripartite entangled for $n=m p q \geq 8$.

Proof. Consider a graph $G=K_{1, n-1}$ on $n=m p q$ vertices, $\left|\alpha_{1}\right\rangle,\left|\alpha_{2}\right\rangle, \cdots,\left|\alpha_{n}\right\rangle$. Then

$$
\rho(G)=\frac{1}{n-1} \sum_{k=2}^{n} \rho\left(H_{1 k}\right)=\frac{1}{n-1} \sum_{k=2}^{n} P\left[\frac{1}{\sqrt{2}}\left(\left|\alpha_{1}\right\rangle-\left|\alpha_{n}\right\rangle\right)\right] .
$$

We are going to examine tripartite separability of $\rho(G)$ in $C_{A}^{m} \otimes C_{B}^{p} \otimes C_{C}^{q}$, where $C_{A}^{m}, C_{B}^{p}$ and $C_{C}^{q}$ are associated to three quantum systems $\mathcal{H}_{A}, \mathcal{H}_{B}$ and $\mathcal{H}_{C}$, respectively. Let $\left\{\left|u_{1}\right\rangle,\left|u_{2}\right\rangle, \cdots,\left|u_{m}\right\rangle\right\},\left\{\left|v_{1}\right\rangle,\left|v_{2}\right\rangle, \cdots,\left|v_{p}\right\rangle\right\}$ and $\left\{\left|w_{1}\right\rangle,\left|w_{2}\right\rangle, \cdots,\left|w_{q}\right\rangle\right\}$ be orthonormal basis of $C_{A}^{m}, C_{B}^{p}$ and $C_{C}^{q}$, respectively. So,

$$
\rho(G)=\frac{1}{n-1} \sum_{k=2}^{n} P\left[\frac{1}{\sqrt{2}}\left(\left|u_{1} v_{1} w_{1}\right\rangle-\left|u_{r_{k}} v_{s_{k}} w_{t_{k}}\right\rangle\right)\right]
$$

where $k=\left(r_{k}-1\right) p q+\left(s_{k}-1\right)+t_{k}, 1 \leq r_{k} \leq m, 1 \leq s_{k} \leq p, 1 \leq t_{k} \leq q$. Hence

$$
\begin{aligned}
\rho(G)= & \frac{1}{n-1}\left\{\sum_{i=2}^{m} P\left[\frac{1}{\sqrt{2}}\left(\left|u_{1}\right\rangle-\left|u_{i}\right\rangle\right)\left|v_{1}\right\rangle\left|w_{1}\right\rangle\right]+\sum_{j=2}^{p} P\left[\left|u_{1}\right\rangle \frac{1}{\sqrt{2}}\left(\left|v_{1}\right\rangle-\left|v_{j}\right\rangle\right)\left|w_{1}\right\rangle\right]\right. \\
& +\sum_{k=2}^{q} P\left[\left|u_{1}\right\rangle\left|v_{1}\right\rangle \frac{1}{\sqrt{2}}\left(\left|w_{1}\right\rangle-\left|w_{k}\right\rangle\right)\right]+\sum_{i=2}^{m} \sum_{j=2}^{p} P\left[\frac{1}{\sqrt{2}}\left(\left|u_{1} v_{1} w_{1}\right\rangle-\left|u_{i} v_{j} w_{1}\right\rangle\right)\right] \\
& +\sum_{j=2}^{p} \sum_{k=2}^{q} P\left[\frac{1}{\sqrt{2}}\left(\left|u_{1} v_{1} w_{1}\right\rangle-\left|u_{1} v_{j} w_{k}\right\rangle\right)\right]+\sum_{i=2}^{m} \sum_{k=2}^{q} P\left[\frac{1}{\sqrt{2}}\left(\left|u_{1} v_{1} w_{1}\right\rangle-\left|u_{i} v_{1} w_{k}\right\rangle\right)\right] \\
& \left.+\sum_{i=2}^{m} \sum_{j=2}^{p} \sum_{k=2}^{q} P\left[\frac{1}{\sqrt{2}}\left(\left|u_{1} v_{1} w_{1}\right\rangle-\left|u_{i} v_{j} w_{k}\right\rangle\right)\right]\right\} .
\end{aligned}
$$

Consider now the following projectors:

$$
P=\left|u_{1}\right\rangle\left\langle u_{1}|+| u_{2}\right\rangle\left\langle u_{2}|, \quad Q=| v_{1}\right\rangle\left\langle v_{1}|+| v_{2}\right\rangle\left\langle v_{2}\right| \text { and } R=\left|w_{1}\right\rangle\left\langle w_{1}|+| w_{2}\right\rangle\left\langle w_{2}\right| .
$$

Then

$$
\begin{aligned}
& (P \otimes Q \otimes R) \rho(G)(P \otimes Q \otimes R) \\
& =\quad \frac{1}{n-1}\left\{\frac{n-8}{2} P\left[\left|u_{1} v_{1} w_{1}\right\rangle\right]+P\left[\frac{1}{\sqrt{2}}\left(\left|u_{1} v_{1} w_{1}\right\rangle-\left|u_{1} v_{1} w_{2}\right\rangle\right)\right]\right. \\
& \quad+P\left[\frac{1}{\sqrt{2}}\left(\left|u_{1} v_{1} w_{1}\right\rangle-\left|u_{1} v_{2} w_{1}\right\rangle\right)\right]+P\left[\frac{1}{\sqrt{2}}\left(\left|u_{1} v_{1} w_{1}\right\rangle-\left|u_{2} v_{1} w_{1}\right\rangle\right)\right] \\
& \quad+P\left[\frac{1}{\sqrt{2}}\left(\left|u_{1} v_{1} w_{1}\right\rangle-\left|u_{1} v_{2} w_{2}\right\rangle\right)\right]+P\left[\frac{1}{\sqrt{2}}\left(\left|u_{1} v_{1} w_{1}\right\rangle-\left|u_{2} v_{1} w_{2}\right\rangle\right)\right] \\
& \left.\quad+P\left[\frac{1}{\sqrt{2}}\left(\left|u_{1} v_{1} w_{1}\right\rangle-\left|u_{2} v_{2} w_{1}\right\rangle\right)\right]+P\left[\frac{1}{\sqrt{2}}\left(\left|u_{1} v_{1} w_{1}\right\rangle-\left|u_{2} v_{2} w_{2}\right\rangle\right)\right]\right\} .
\end{aligned}
$$

In the basis

$$
\left\{\left|u_{1} v_{1} w_{1}\right\rangle,\left|u_{1} v_{1} w_{2}\right\rangle,\left|u_{1} v_{2} w_{1}\right\rangle,\left|u_{1} v_{2} w_{2}\right\rangle,\left|u_{2} v_{1} w_{1}\right\rangle,\left|u_{2} v_{1} w_{2}\right\rangle,\left|u_{2} v_{2} w_{1}\right\rangle,\left|u_{2} v_{2} w_{2}\right\rangle\right\}
$$


we have

$$
[(P \otimes Q \otimes R) \rho(G)(P \otimes Q \otimes R)]^{T_{A}}=\frac{1}{n-1}\left(\begin{array}{cccccccc}
\frac{n-1}{2} & -\frac{1}{2} & -\frac{1}{2} & -\frac{1}{2} & -\frac{1}{2} & 0 & 0 & 0 \\
-\frac{1}{2} & \frac{1}{2} & 0 & 0 & -\frac{1}{2} & 0 & 0 & 0 \\
-\frac{1}{2} & 0 & \frac{1}{2} & 0 & -\frac{1}{2} & 0 & 0 & 0 \\
-\frac{1}{2} & 0 & 0 & \frac{1}{2} & -\frac{1}{2} & 0 & 0 & 0 \\
-\frac{1}{2} & -\frac{1}{2} & -\frac{1}{2} & -\frac{1}{2} & \frac{1}{2} & 0 & 0 & 0 \\
0 & 0 & 0 & 0 & 0 & \frac{1}{2} & 0 & 0 \\
0 & 0 & 0 & 0 & 0 & 0 & \frac{1}{2} & 0 \\
0 & 0 & 0 & 0 & 0 & 0 & 0 & \frac{1}{2}
\end{array}\right) .
$$

The eigenpolynomial of the above matrix is

$$
\left(\lambda-\frac{1}{2(n-1)}\right)^{5}\left(\lambda^{3}-\frac{n+1}{2(n-1)} \lambda^{2}+\frac{n-4}{2(n-1)^{2}} \lambda+\frac{n+4}{4(n-1)^{3}}\right)
$$

so the eigenvalues of the matrix are $\frac{1}{2(n-1)}$ (with multiplicity 5 ) and the roots of the polynomial $\lambda^{3}-\frac{n+1}{2(n-1)} \lambda^{2}+\frac{n-4}{2(n-1)^{2}} \lambda+\frac{n+4}{4(n-1)^{3}}$. Let the roots of this polynomial of degree three be $\lambda_{1}, \lambda_{2}$ and $\lambda_{3}$. Then $\lambda_{1} \lambda_{2} \lambda_{3}=-\frac{n+4}{4(n-1)^{3}}<0$, so one of the three roots must be negative, i.e., there must be a negative eigenvalue of the above matrix. Hence, by Peres criterion, the matrix $(P \otimes Q \otimes R) \rho(G)(P \otimes Q \otimes R)$ is tripartite entangled and then $\rho(G)$ is tripartite entangled.

\section{A sufficient and necessary condition of tripartite separability}

Definition 2 Partially transposed graph $G^{\Gamma_{A}}=\left(V, E^{\prime}\right)$, (i.e. the partial transpose of a graph $G=(V, E)$ with respect to $\left.\mathcal{H}_{A}\right)$ is the graph such that

$$
\left\{u_{i} v_{j} w_{k}, u_{r} v_{s} w_{t}\right\} \in E^{\prime} \text { if and only if }\left\{u_{r} v_{j} w_{k}, u_{i} v_{s} w_{t}\right\} \in E .
$$

Partially transposed graphs $G^{\Gamma_{B}}$ and $G^{\Gamma_{C}}$ (with respect to $\mathcal{H}_{B}$ and $\mathcal{H}_{C}$, respectively) can be defined in a similar way.

For tripartite states we denote $\Delta(G)=\Delta\left(G^{\Gamma_{A}}\right)=\Delta\left(G^{\Gamma_{B}}\right)=\Delta\left(G^{\Gamma_{C}}\right)$ as the degree condition. Hildebrand et al. ${ }^{[12]}$ proved that the degree criterion is equivalent to PPT criterion. It is easy to show that this equivalent condition is still true for the tripartite states. Thus from Peres criterion we can get:

Theorem 3 Let $\rho(G)$ be the density matrix of a graph on $n=m p q$ vertices. If $\rho(G)$ is separable in $C_{A}^{m} \otimes C_{B}^{p} \otimes C_{C}^{q}$, then $\Delta(G)=\Delta\left(G^{\Gamma_{A}}\right)=\Delta\left(G^{\Gamma_{B}}\right)=\Delta\left(G^{\Gamma_{C}}\right)$. 
Let $G$ be a graph on $n=m p q$ vertices: $\alpha_{1}, \alpha_{2}, \cdots, \alpha_{n}$ and $f$ edges: $\left\{\alpha_{i_{1}}, \alpha_{j_{1}}\right\}$, $\left\{\alpha_{i_{2}}, \alpha_{j_{2}}\right\}, \cdots,\left\{\alpha_{i_{f}}, \alpha_{j_{f}}\right\}$. Let vertices $\alpha_{s}=u_{i} v_{j} w_{k}$, where $s=(i-1) p q+(j-1) q+k, 1 \leq$ $i \leq m, 1 \leq j \leq p, 1 \leq k \leq q$. The vectors $\left|u_{i}\right\rangle^{\prime} s,\left|v_{j}\right\rangle^{\prime} s,\left|w_{k}\right\rangle^{\prime} s$ form orthonormal bases of $C^{m}, C^{p}$ and $C^{q}$, respectively. The edge $\left\{u_{i} v_{j} w_{k}, u_{r} v_{s} w_{t}\right\}$ is said to be entangled if $i \neq r, j \neq s, k \neq t$.

Consider a cuboid with $m p q$ points whose length is $m$, width is $p$ and height is $q$, such that the distance between two neighboring points on the same line is 1. A nearest point graph is a graph whose vertices are identified with the points of the cuboid and the edges have length $1, \sqrt{2}$ and $\sqrt{3}$.

The degree condition is still a sufficient condition of the tripartite separability for the density matrix of a nearest point graph.

Theorem 4 Let $G$ be a nearest point graph on $n=m p q$ vertices. If $\Delta(G)=$ $\Delta\left(G^{\Gamma_{A}}\right)=\Delta\left(G^{\Gamma_{B}}\right)=\Delta\left(G^{\Gamma_{C}}\right)$, then the density matrix $\rho(G)$ is tripartite separable in $C_{A}^{m} \otimes C_{B}^{p} \otimes C_{C}^{q}$

Proof. Let $G$ be a nearest point graph on $n=m p q$ vertices and $f$ edges. We associate to $G$ the orthonormal basis $\left\{\left|\alpha_{l}\right\rangle: l=1,2, \cdots, n\right\}=\left\{\left|u_{i}\right\rangle \otimes\left|v_{j}\right\rangle \otimes\left|w_{k}\right\rangle: i=\right.$ $1,2, \cdots, m ; j=1,2, \cdots, p ; k=1,2, \cdots, q\}$, where $\left\{\left|u_{i}\right\rangle: i=1,2, \cdots, m\right\}$ is an orthonormal basis of $C_{A}^{m},\left\{\left|v_{j}\right\rangle: j=1,2, \cdots, p\right\}$ is an orthonormal basis of $C_{B}^{p}$ and $\left\{\left|w_{k}\right\rangle: i=1,2, \cdots, q\right\}$ is an orthonormal basis of $C_{C}^{q}$. Let $i, r \in\{1,2, \cdots, m\}, j, s \in$ $\{1,2, \cdots, p\}, k, t \in\{1,2, \cdots, q\}, \lambda_{i j k, r s t} \in\{0,1\}$ be defined by

$$
\lambda_{i j k, r s t}= \begin{cases}1, & \text { if }\left(u_{i} v_{j} w_{k}, u_{r} v_{s} w_{t}\right) \in E(G) \\ 0, & \text { if }\left(u_{i} v_{j} w_{k}, u_{r} v_{s} w_{t}\right) \notin E(G)\end{cases}
$$

where $i, j, k, r, s, t$ satisfy either of the following seven conditions:

- $i=r, j=s, k=t+1$;

- $i=r, j=s+1, k=t$;

- $i=r+1, j=s, k=t$

- $i=r, j=s+1, k=t+1$;

- $i=r+1, j=s+1, k=t$;

- $i=r+1, j=s, k=t+1$;

- $i=r+1, j=s+1, k=t+1$.

Let $\rho(G), \rho\left(G^{\Gamma_{A}}\right), \rho\left(G^{\Gamma_{B}}\right)$ and $\rho\left(G^{\Gamma_{C}}\right)$ be the density matrices corresponding to the graph $G, G^{\Gamma_{A}}, G^{\Gamma_{B}}$ and $G^{\Gamma_{C}}$, respectively. Thus

$$
\begin{array}{rlrl}
\rho(G) & =\frac{1}{2 f}(\Delta(G)-M(G)), & & \rho\left(G^{\Gamma_{A}}\right)=\frac{1}{2 f}\left(\Delta\left(G^{\Gamma_{A}}\right)-M\left(G^{\Gamma_{A}}\right)\right), \\
\rho\left(G^{\Gamma_{B}}\right) & =\frac{1}{2 f}\left(\Delta\left(G^{\Gamma_{B}}\right)-M\left(G^{\Gamma_{B}}\right)\right), & \rho\left(G^{\Gamma_{C}}\right)=\frac{1}{2 f}\left(\Delta\left(G^{\Gamma_{C}}\right)-M\left(G^{\Gamma_{C}}\right)\right) .
\end{array}
$$


Let $G_{1}$ be the subgraph of $G$ whose edges are all the entangled edges of $G$. An edge $\left\{u_{i} v_{j} w_{k}, u_{r} v_{s} w_{t}\right\}$ is entangled if $i \neq r, j \neq s, k \neq t$. Let $G_{1}^{A}$ be the subgraph of $G^{\Gamma_{A}}$ corresponding to all the entangled edges of $G^{\Gamma_{A}}, G_{1}^{B}$ be the subgraph of $G^{\Gamma_{B}}$ corresponding to all the entangled edges of $G^{\Gamma_{B}}$, and $G_{1}^{C}$ be the subgraph of $G^{\Gamma_{C}}$ corresponding to all the entangled edges of $G^{\Gamma_{C}}$. Obviously, $G_{1}^{A}=\left(G_{1}\right)^{\Gamma_{A}}, G_{1}^{B}=\left(G_{1}\right)^{\Gamma_{B}}, G_{1}^{C}=\left(G_{1}\right)^{\Gamma_{C}}$. We have

$$
\rho\left(G_{1}\right)=\frac{1}{f} \sum_{i=1}^{m} \sum_{j=1}^{p} \sum_{k=1}^{q} \lambda_{i j k, r s t} P\left[\frac{1}{\sqrt{2}}\left(\left|u_{i} v_{j} w_{k}\right\rangle-\left|u_{r} v_{s} w_{t}\right\rangle\right)\right],
$$

where $i, j, k ; r, s, t$ must satisfy either of the above seven conditions. We can get $\rho\left(G_{1}^{A}\right), \rho\left(G_{1}^{B}\right)$ and $\rho\left(G_{1}^{C}\right)$ by commuting the index of $u, v, w$ in the above equation, respectively. Also we have

$$
\Delta\left(G_{1}\right)=\frac{1}{2 f} \sum_{i=1}^{m} \sum_{j=1}^{p} \sum_{k=1}^{q} \lambda_{i j k, r s t} P\left[\left|u_{i} v_{j} w_{k}\right\rangle\right]
$$

where $i, j, k ; r, s, t$ must satisfy either of the above seven conditions. We can get $\Delta\left(G_{1}^{A}\right), \Delta\left(G_{1}^{B}\right)$ and $\Delta\left(G_{1}^{C}\right)$ by commuting the index of $\lambda$ with respect to the Hilbert space $\mathcal{H}_{A}, \mathcal{H}_{B}, \mathcal{H}_{C}$, respectively. Let $G_{2}, G_{2}^{A}, G_{2}^{B}$ and $G_{2}^{C}$ be the subgraph of $G, G^{A}, G^{B}$ and $G^{C}$ containing all the unentangled edges, respectively. It is obvious that $\Delta\left(G_{2}\right)=$ $\Delta\left(G_{2}^{\Gamma_{A}}\right)=\Delta\left(G_{2}^{\Gamma_{B}}\right)=\Delta\left(G_{2}^{\Gamma_{C}}\right)$. So $\Delta(G)=\Delta\left(G^{\Gamma_{A}}\right)=\Delta\left(G^{\Gamma_{B}}\right)=\Delta\left(G^{\Gamma_{C}}\right)$ if and only if $\Delta\left(G_{1}\right)=\Delta\left(G_{1}^{\Gamma_{A}}\right)=\Delta\left(G_{1}^{\Gamma_{B}}\right)=\Delta\left(G_{1}^{\Gamma_{C}}\right)$. The degree condition implies that

$$
\lambda_{i j k, r s t}=\lambda_{r j k, i s t}=\lambda_{i s k, r j t}=\lambda_{i j t, r s k},
$$

for any $i, r \in\{1,2, \cdots, m\}, j, s \in\{1,2, \cdots, p\}, k, t \in\{1,2, \cdots, q\}$. The above equation shows that whenever there is an entangled edge $\left\{u_{i} v_{j} w_{k}, u_{r} v_{s} w_{t}\right\}$ in $G$ (here we must have $i \neq r, j \neq s, k \neq t$ ), there must be the entangled edges $\left\{u_{r} v_{j} w_{k}, u_{i} v_{s} w_{t}\right\},\left\{u_{i} v_{s} w_{k}, u_{r} v_{j} w_{t}\right\}$ and $\left\{u_{i} v_{j} w_{t}, u_{r} v_{s} w_{k}\right\}$ in $G$. Let

$$
\begin{aligned}
\rho(i, j, k ; r, s, t)= & \frac{1}{4}\left(P\left[\frac{1}{\sqrt{2}}\left(\left|u_{i} v_{j} w_{k}\right\rangle-\left|u_{r} v_{s} w_{t}\right\rangle\right)\right]+P\left[\frac{1}{\sqrt{2}}\left(\left|u_{r} v_{j} w_{k}\right\rangle-\left|u_{i} v_{s} w_{t}\right\rangle\right)\right]\right. \\
& \left.+P\left[\frac{1}{\sqrt{2}}\left(\left|u_{i} v_{s} w_{k}\right\rangle-\left|u_{r} v_{j} w_{t}\right\rangle\right)\right]+P\left[\frac{1}{\sqrt{2}}\left(\left|u_{i} v_{j} w_{t}\right\rangle-\left|u_{r} v_{s} w_{k}\right\rangle\right)\right]\right) .
\end{aligned}
$$

By Lemma 2, we know $\rho(i, j, k ; r, s, t)$ is tripartite separable in $C_{A}^{m} \otimes C_{B}^{p} \otimes C_{C}^{q}$. By Theorem 3 in [11] we can easily get $\rho\left(G_{2}\right)$ is tripartite separable in $C_{A}^{m} \otimes C_{B}^{p} \otimes C_{C}^{q}$.

From Theorems 3 and 4 we can obtain the following corollary which is a sufficient and necessary criterion (we called degree-criterion) of the density matrix of a nearest point graph:

Corollary 1 Let $G$ be a nearest point graph on $n=m p q$ vertices, then the density matrix $\rho(G)$ is tripartite separable in $C_{A}^{m} \otimes C_{B}^{p} \otimes C_{C}^{q}$ if and only if $\Delta(G)=\Delta\left(G^{\Gamma_{A}}\right)=$ $\Delta\left(G^{\Gamma_{B}}\right)=\Delta\left(G^{\Gamma_{C}}\right)$. 
Example Let $G$ be a graph on $12=3 \times 2 \times 2$ vertices, having a unique edge $\left\{u_{1} v_{1} w_{1}, u_{2} v_{2} w_{2}\right\}$. Then we have

$$
\rho(G)=\frac{1}{2}\left(\begin{array}{cccccccccccc}
1 & 0 & 0 & 0 & 0 & 0 & 0 & -1 & 0 & 0 & 0 & 0 \\
0 & 0 & 0 & 0 & 0 & 0 & 0 & 0 & 0 & 0 & 0 & 0 \\
0 & 0 & 0 & 0 & 0 & 0 & 0 & 0 & 0 & 0 & 0 & 0 \\
0 & 0 & 0 & 0 & 0 & 0 & 0 & 0 & 0 & 0 & 0 & 0 \\
0 & 0 & 0 & 0 & 0 & 0 & 0 & 0 & 0 & 0 & 0 & 0 \\
0 & 0 & 0 & 0 & 0 & 0 & 0 & 0 & 0 & 0 & 0 & 0 \\
0 & 0 & 0 & 0 & 0 & 0 & 0 & 0 & 0 & 0 & 0 & 0 \\
-1 & 0 & 0 & 0 & 0 & 0 & 0 & 1 & 0 & 0 & 0 & 0 \\
0 & 0 & 0 & 0 & 0 & 0 & 0 & 0 & 0 & 0 & 0 & 0 \\
0 & 0 & 0 & 0 & 0 & 0 & 0 & 0 & 0 & 0 & 0 & 0 \\
0 & 0 & 0 & 0 & 0 & 0 & 0 & 0 & 0 & 0 & 0 & 0 \\
0 & 0 & 0 & 0 & 0 & 0 & 0 & 0 & 0 & 0 & 0 & 0
\end{array}\right) .
$$

The partially transposed graph $G^{\Gamma_{A}}$ is a graph on 12 vertices and has an edge $\left\{u_{2} v_{1} w_{1}\right.$, $\left.u_{1} v_{2} w_{2}\right\}$. Then

$$
\rho\left(G^{\Gamma_{A}}\right)=\frac{1}{2}\left(\begin{array}{cccccccccccc}
0 & 0 & 0 & 0 & 0 & 0 & 0 & 0 & 0 & 0 & 0 & 0 \\
0 & 0 & 0 & 0 & 0 & 0 & 0 & 0 & 0 & 0 & 0 & 0 \\
0 & 0 & 0 & 0 & 0 & 0 & 0 & 0 & 0 & 0 & 0 & 0 \\
0 & 0 & 0 & 1 & -1 & 0 & 0 & 0 & 0 & 0 & 0 & 0 \\
0 & 0 & 0 & -1 & 1 & 0 & 0 & 0 & 0 & 0 & 0 & 0 \\
0 & 0 & 0 & 0 & 0 & 0 & 0 & 0 & 0 & 0 & 0 & 0 \\
0 & 0 & 0 & 0 & 0 & 0 & 0 & 0 & 0 & 0 & 0 & 0 \\
0 & 0 & 0 & 0 & 0 & 0 & 0 & 0 & 0 & 0 & 0 & 0 \\
0 & 0 & 0 & 0 & 0 & 0 & 0 & 0 & 0 & 0 & 0 & 0 \\
0 & 0 & 0 & 0 & 0 & 0 & 0 & 0 & 0 & 0 & 0 & 0 \\
0 & 0 & 0 & 0 & 0 & 0 & 0 & 0 & 0 & 0 & 0 & 0 \\
0 & 0 & 0 & 0 & 0 & 0 & 0 & 0 & 0 & 0 & 0 & 0
\end{array}\right) .
$$

Obviously, the degree matrices of $G$ and $G^{\Gamma_{A}}$ are different. The eigenvalues of $\rho(G)^{T_{A}}$ are 0 (with multiplicity 8 ), $\frac{1}{2}$ (with multiplicity 3 ) and $-\frac{1}{2}$, so $\rho(G)^{T_{A}}$ is not positive semidefinite. According to Peres criterion, $\rho(G)$ is tripartite entangled.

Two graphs $G$ and $H$ are said to be isomorphic, denoted as $G \cong H$, if there is an isomorphism between $V(G)$ and $V(H)$, i.e., there is a permutation matrix $P$ such that $P M(G) P^{T}=M(H) \cdot{ }^{[8]}$

Theorem 5 Let $G$ and $H$ be two graphs on $n=m p q$ vertices. If $\rho(G)$ is tripartite entangled in $C^{m} \otimes C^{p} \otimes C^{q}$ and $G \cong H$, then $\rho(H)$ is not necessarily tripartite entangled in $C^{m} \otimes C^{p} \otimes C^{q}$.

Proof. Let $G$ be the graph introduced in the above example. Then $\rho(G)$ is tripartite entangled. Let $H$ be a graph on 12 vertices, having an edge $\left\{u_{1} v_{1} w_{1}, u_{1} v_{1} w_{2}\right\}$. Obviously, $G$ is isomorphic to $H$. However,

$$
\rho(H)=P\left[\frac{1}{\sqrt{2}}\left(\left|u_{1} v_{1} w_{1}\right\rangle-\left|u_{1} v_{1} w_{2}\right\rangle\right)\right]=\left|u_{1}\right\rangle\left\langle u_{1}|\otimes| v_{1}\right\rangle\left\langle v_{1}|\otimes| w^{+}\right\rangle\left\langle w^{+}\right|,
$$


where $\left|w^{+}\right\rangle=\frac{1}{\sqrt{2}}\left(\left|w_{1}\right\rangle-\left|w_{2}\right\rangle\right)$, shows that $\rho(H)$ is tripartite separable.

\section{References}

[1] A. Peres, Quantum Theory: Concepts and Methods Kluwer, Dordrecht 1995.

[2] A. Ekert, Phys. Rev. Lett. 67 (1991) 661.

[3] D. Deutsch, Proc. R. Soc. London, Ser. A 425 (1989) 73.

P. Shor, SIAM J. Comput. 26 (1997) 1484.

[4] C. H. Bennett and S. J. Wiesner, Phys. Rev. Lett. 69 (1992) 2881.

[5] K. Mattle, H. Weinfurter, P. Kwiat and A. Zeilinger, Phys. Rev. Lett. 76 (1996) 4656.

[6] C. Bennett, G. Brassard, C. Crepeau, R. Jozsa, A. Peres and W. K. Wootters, Phys. Rev. Lett. 70 (1993) 1895.

[7] D. Bouwmeester, J. W. Pan, K. Mattle, M. Elbl, H. Weinfurer and A. Zeilinger, Nature (London) 390 (1997) 575.

D. Boschi, S. Branca, F. de Martini, L. Hardy and S. Popescu, Phys. Rev. Lett. 80 (1998) 1121.

[8] S. L. Braunstein, S. Ghosh, S. Severini, Annals of Combinatorics 10 (2006) 291.

[9] Ali Saif M. Hassan and P. Joag, Combinatorial approach to multipartite quantum system: basic formulation, arXiv: quant-ph/0602053v5.

[10] Chai Wah Wu, Phys. Lett. A 351 (2006) 18.

[11] S. L. Braunstein, S. Ghosh, T. Mansour, S. Severini and R. C. Wilson, Phys. Rev. A 73 (2006) 012320.

[12] R. Hildebrand, S. Mancini and S. Severini, Combinatorial Laplacian and Positivity Under Partial Transpose, arXiv: cs. CC/0607036, Accepted in Mathematical Structure in Computer Scinence.

[13] A. Peres, Phys. Rev. Lett. 77 (1996) 1413.

K. Życzkowski, P. Horodecki and R. Horodecki, Phys. Lett. A 223 (1996) 1.

[14] C. Godsil and G. Royle, Algebratic Graph Theory, Graduate Texts in Mathematics, 207, Springer-Verlag, New York, 2001. 